\title{
Fiber Bundle Description of Flow and Nonlinear Hydrodynamics on Circles
}

\author{
Andrei LUDU \\ Northwestern State University, Dept. Chemistry and Physics \\ Natchitoches, LA 71497, USA \\ and \\ University Antwerp, Dept. Physics, CMTG, Antwerp, B-2020, Belgium \\ E-mail: ludua@nsula.edu
}

Received on April 4, 2008, revised on May 6, 2008

\begin{abstract}
We introduce a differential geometry description of the path lines, stream lines and particles contours in hydrodynamics. We present a generalized form of a Korteweg-de Vries type of equation for the exterior of a circle. Nonlinearities from the boundary conditions, surface tension and the Euler equations are taken into account, but the flow is considered inviscid and irrotational. For the circular case we describe the traveling waves shapes, solitons and the particles trajectories.
\end{abstract}

\section{Introduction: path lines, stream lines and particles con- tours}

We introduce the working space $(t, \vec{r}) \in \mathbf{R} \times \mathbf{R}^{3}$. From the Lagrangian point of view the fluid particle motions are nonintersecting regular curves $\Gamma_{L}$ in this base space, parametrized by time and described by equations $\vec{r}_{L}\left(t, \vec{r}_{0}\right)$. They are called paths, or material lines, [1], or lines of motion [2]. Since they do not intersect, each such curve is labeled by one of its points, $\vec{r}_{0}$, for example the position of the particle when $t=0$. The tangent to this curve is

$$
\vec{t}_{L}=\left(1, \vec{v}_{L}\right)\left(1+v_{L}^{2}\right)^{-1 / 2},
$$

where $\vec{v}_{L}=\partial \vec{r}_{L}\left(t, \vec{r}_{0}\right) / \partial t$ is the Lagrangian velocity of the particle along the path. All these paths do not intersect and completely fill the base space when $\vec{r}_{0} \in \mathbf{R}^{3}$.

If we choose a fixed point in space $\vec{r}$, some of the paths $\vec{r}_{0}$ will intersect this fixed point, $\vec{r}_{L}\left(t, \vec{r}_{0}\right)=\vec{r}$, so that we can write the "list" of these particles versus time: $\vec{r}_{0}=\vec{r}_{0}(t, \vec{r})$. We define the Eulerian velocity at $(t, \vec{r})$ by substituting this $\vec{r}_{0}(t, \vec{r})$ list in the velocity expression

$$
\vec{v}_{E}(t, \vec{r})=\vec{v}_{L}\left(t, \vec{r}_{0}(t, \vec{r})\right) .
$$

If we need a physical quantity $Q$, defined for any fluid particle, for the particle labeled by $\vec{r}_{0}$, the Lagrangian value $Q_{L}\left(t, \vec{r}_{0}\right)$ is defined along $\Gamma_{L}$. Suppose this $\Gamma_{L}$ intersects a 
fixed line $\vec{r}=$ const. at $\vec{r}_{L}\left(t, \vec{r}_{0}\right)=\vec{r}$. By solving this equation with respect to $\vec{r}_{0}$, we have $\vec{r}_{0}=\vec{r}_{0}(t, \vec{r})$. We can define now the Eulerian value of $Q$ by

$$
Q_{E}(t, \vec{r})=Q_{L}\left(t, \vec{r}_{0}(t, \vec{r})\right) .
$$

While following the particle in its motion, the quantity $Q_{L}$ has a variation $d Q_{L}\left(t, \vec{r}_{0}\right)=$ $\left(d Q_{L} / d t\right) d t$. At $\vec{r}=$ const. the quantity $Q_{E}$ has another variation $d Q_{E}=\left(\partial Q_{E} / \partial t\right) d t$. By differentiation of Eq. (1.2) we have

$$
d Q_{L}=d Q_{E}+\left(d \vec{r}_{L} \cdot \nabla Q_{E}\right) d t
$$

Since we follow the particle in its motion we have $d \vec{r}_{L}=\vec{v}_{L} d t$.

\section{The fluid fiber bundle}

We introduce the available space for the fluid (the reference fluid container [3],[4]) as a domain $D$ of $\mathbf{R}^{3}$, and add an extra dimension for time to form a base space $D \times \mathbf{R}$. The particle paths $\vec{r}_{L}\left(\vec{r}_{0}, t\right)$ are smooth time-parametrized curves in this base space. The projection on the horizontal planes (projections perpendicular on the time axis) of the tangent vectors to these curves represent the velocity fields of the particles. The two velocities, i.e. the Lagrangian (material) and Eulerian (spatial) velocities, have the same value at the same point of the base space. The only difference between these two types of velocities consists in the parametrization of the vector fields. The Lagrangian velocity field is defined along the particle paths in the base space, while the Eulerian velocity field is defined on the horizontal plane, in points where these paths intersect it, at a moment of time $t$. The integral curves of the Eulerian velocity field contained in any "horizontal" plane are the stream lines at that moment of time. However, the paths lines do not identify with the lift of the stream lines in the base space. Namely, if we choose a point $\vec{r}$ in some horizontal plane $t$ and we compare the path line crossing through this point, and the vertical lift of the stream line crossing the same point, these two curves are different in general. An example is presented in Fig.1. For any given fixed point $\vec{r}_{0}$ in the initial plane we can draw all paths crossing this at different moments of time, Fig. 2. The intersections of all these paths with a certain horizontal plane $t$ generate a streak line initiated by a "nozzle" placed at $\vec{r}_{0}$. In traditional approaches the motion of the particles is described by a one-parameter (time) group of diffeomorphisms acting on the domain $D\left(\vec{r}_{0}\right)$. The Lagrange coordinate of a particle is the result of the action of this group on the corresponding element $\vec{r}_{0}$. If the motion is incompressible, the group of diffeomorphisms is volume preserving. In this formalism, the infinitesimal generator of the group is the Lagrangian field of velocities.

However, such a model is not exact. That is because it associates the same geometrical space to physical spaces with different signification, namely the material points (initial positions space), and the spatial points per se. Even if initially $(t=0)$ the positions $\vec{r}_{0}$ of all fluid particles, $\vec{r}_{0} \in D$, belong the a position space, during the motion these vectors actually form a space of parameters, labeling the particles. On the other hand, the positions of the particles at any arbitrary moment of time (given by the Lagrangian equations of motion $\left.\vec{r}_{L}\left(\vec{r}_{0}, t\right)\right)$ belong to a space of positions. 


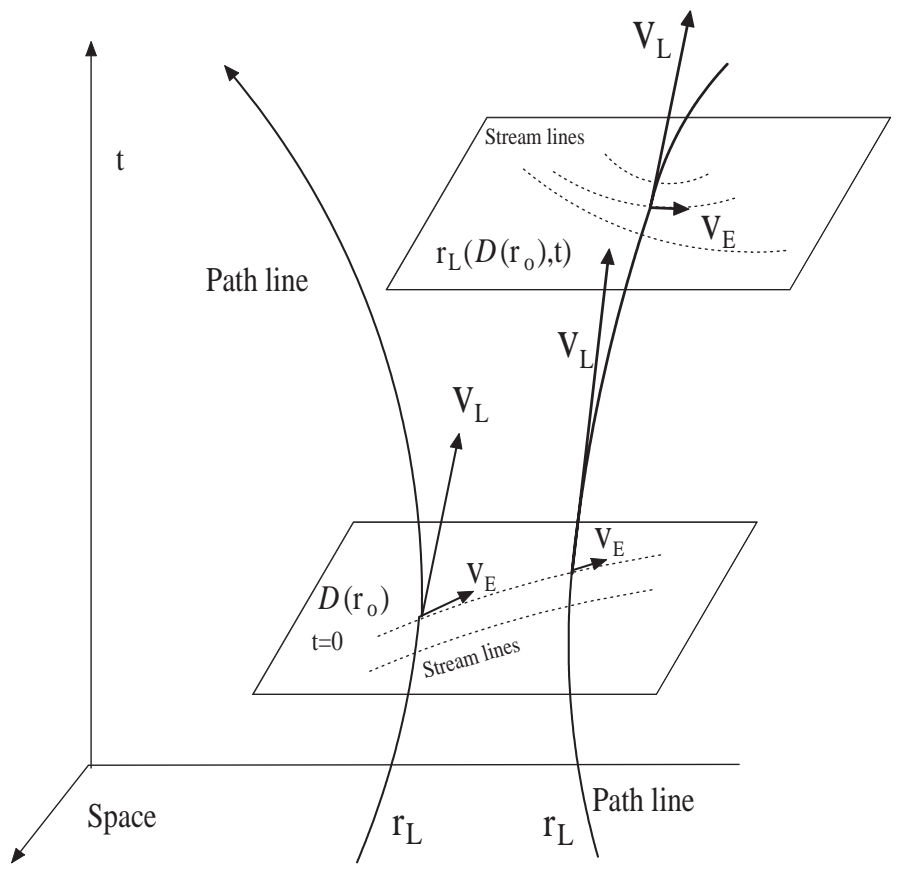

Figure 1: A 2-dimensional fluid domain $D\left(\vec{r}_{0}\right)$ and two path lines $\vec{r}_{L}(t)$ whose tangents are the Lagrangian velocities $\vec{v}_{L}$. The projection of the Lagrangian velocity field on the tangent space of the fluid domain is the Eulerian velocity field $\vec{v}_{\mathcal{E}}$. The integral curves of the Eulerian vector field in the fluid domain, at a given moment of time $t$, are the stream lines at that moment (dotted lines).

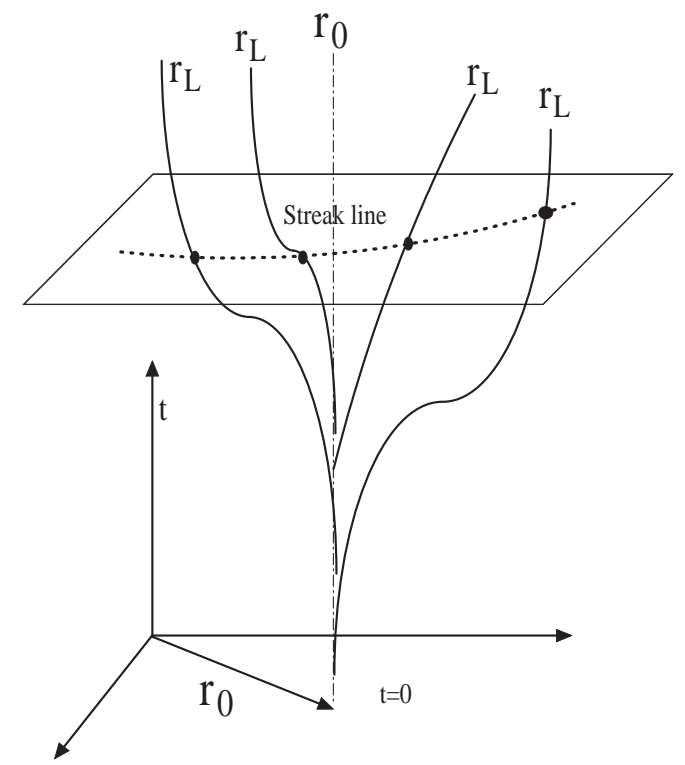

Figure 2: Paths emerging from a nozzle point $\vec{r}_{0}$ (dashed-dotted axis) at different moments of time. The intersections of all such paths with a horizontal plane $t$ provide a streak line (dotted). 
We present a formalism in which a fluid is described using cross-sections $\sigma$ in a fiber bundle $\mathcal{F}$ over some base manifold $X$. In the case of a fixed container for the fluid (even the case of the whole space) we consider the base to be the space of particles (usually labeled by their initial positions) and the fiber is the space available for particles positions. On the contrary, a free surface introduces one more freedom in the problem. We cannot construct using the same pattern because we allow different particles to belong to different shapes simultaneously, which is impossible. In order to build a fiber bundle in this case we use a formalism from the mechanics of deformable bodies. The base space is the manifold of all possible shapes, and the standard fiber is particle position space. The role of the particle labeling space is taken over by the nontrivial structure group.

The base manifold is usually a space-time manifold built as a product between a smooth 3 -dimensional oriented Riemannian manifold $(M, g)$, where $g$ is the metric, and $\mathbf{R}$ for time, i.e. $X=M \times \mathbf{R}$. The coordinates in $X$ are $x=\left(x^{\mu}\right)=\left(x^{i}, t\right) \in X$, with $i=1, \ldots, 3, \mu=$ $1, \ldots, 4$. For fluid dynamics we can choose the fiber $F=M$ with coordinates $y \in F[3]$. Consequently, the local coordinates in this $\mathcal{F}$ bundle over $X$ are $(x, t, y)$ and the projection is $\Pi: \mathcal{F} \rightarrow X, \quad(x, t, y) \rightarrow(x, t)$. Transformations and operations that affect only the base (spatial changes like rotations, etc.) are called fiber-preserving transformations. A lift of any geometrical object $\gamma$ (a curve, surface, function, form, etc.) defined in the base space, is a map of this object into the fiber bundle, $\gamma \rightarrow \gamma^{\prime} \in \mathcal{F}$, such that it projects back down to the original object in $M, \Pi \circ \gamma^{\prime}=\gamma$.

Cross-sections in this bundle $\sigma: X \rightarrow \mathcal{F}$ represent time dependent configurations, i.e. particle position fields. The cross-section has the coordinates $\sigma(x)=\left(x^{\mu}, \sigma^{i}(x)\right)=\left(x^{\mu}, y^{i}\right)$. On the top of the configuration bundle $\mathcal{E}$ we can construct another fiber bundle $J^{1} \mathcal{F}$ over $\mathcal{F}$ called the first jet bundle, $[3,5]$, with the fiber above $(x, y)$ consisting of linear maps from the tangent space of the base space to the tangent space of the bundle, $\gamma: T_{x} X \rightarrow T_{(x, y)} \mathcal{F}$, satisfying $d \pi \circ \gamma=I d_{T_{x} X}$.

For any cross-section $\sigma$ in $\mathcal{F}$ over $X$, the differential $d \sigma_{x}$ at $x$ is an element of the jet bundle $J^{1} \mathcal{F}_{\sigma(x)}$. Consequently, the map $x \rightarrow d \sigma_{x}$ is a cross-section of the jet bundle over $X$. This section, denoted $j^{1} \sigma$ is called the first jet extension of $\sigma$. In coordinates, it is given by $j^{1} \sigma(x)=\left(x^{\mu}, \sigma^{i}(x), \partial_{\mu} \sigma^{i}\right)$, where $\partial_{\mu}=\left(\partial_{i}, \partial_{t}\right)$. It is this triple which represents the fluid motion. The first three base coordinates space components $x^{i}$, originally coming from the initial positions of the fluid particles, now represent the particle labeling. The $\sigma^{i}(x)$ components identify the position of the $x$ particle in space, and the $\partial_{t} \sigma^{i}$ components represent the velocity of the particle $x$.

For the case when the fluid moves in a fixed region, i.e. with fixed boundaries, the group structure of the fiber bundle $\mathcal{F}$ is the identity, and the bundle is trivial, $\mathcal{F}=X \times M$. The spatial part of the base manifold $M$ represents the reference configuration (initial positions of all fluid particles). Actually, the coordinate $x$ ceases to represent the initial position, but remains attached to the particle and labels it for the rest of the evolution. So, the space part of the base manifold $x$ (the material points) labels the fluid particles through the one-to-one correspondence between particles and their initial positions in the reference fluid container. The time base $X$ corresponds to the time evolution. The fiber over any base point is the same manifold, meaning that the space available for any particle is the same at any moment of time. Its coordinates $y$ are called $F_{(x, t)}$ represents the available space for particle $x$ at the moment $t$, and it is diffeomorphic with $M$, i.e. the reference fluid container $[3,4]$. In the case of $\mathcal{F}$, the requirement for the existence of a projection 
$\Pi: \mathcal{F} \rightarrow X$ from the definition of a fiber bundle guaranties that all points of the fiber, at any point of the base, are filled with fluid.

The fluid motion is described by a cross-section $\sigma(x, t)$ of the bundle $\mathcal{F}$ representing the particle placement field. Not any cross-section can represent a real motion of the fluid, and some minimal constrains are needed. First, $\sigma$ is not allowed to create or annihilate fluid particles, and second, two different particles cannot hold the same spatial point at the same moment of time. These two constrains are fulfilled by requesting that the Lagrangian paths of the fluid particles represent a diffeomorphism of the reference fluid container. In the fiber bundle formalism these two physical constraint require a similar thing. The restriction of the cross section $\left.\sigma(x, t)\right|_{t=t_{0}}$ at a constant $t=t_{0}$ (for every moment of time $t_{0}$ ) needs to be a diffeomorphism of the manifold $F=M$. Of course, this is also possible because the bundle is trivial, and there is a canonical diffeomorphism between any two fibers at any two points.

In the local coordinates of a given fiber, $y(x, t) \in F_{(x, t)}$ represents the spatial position of the particle $x$ at moment $t,(x, t, y) \sigma(x, t)$. The path lines are the restrictions of the crosssection $\vec{r}_{L}\left(x_{0}, t\right)=\left.\sigma\right|_{x=\left(x_{0}, t\right)}$ for fixed point in the space part of the base space. The tangent vectors to these curves can be expressed in two ways. If we write $\vec{v}_{L}(x, t)=\partial \sigma^{v}(x, t) / \partial t$ we have the Lagrangian (material) velocity field. The superscript $v$ (as in vertical) represents the components of the cross-section along the fiber. The Lagrangian velocity field is actually represented by the last three components of the cross-section in the first jet bundle $d \sigma$. Namely $j^{1} \sigma=\left(\sigma, \partial_{i} \sigma, \vec{v}_{L}\right)$.

Conversely, if we invert the equation $y(x, t)$ with respect to $y$, we can express the velocity field in coordinates $\vec{v}_{L}(x(y), t)=\vec{v}_{E}(y, t)$, which is nothing but the Eulerian velocity field. So, even if locally the Eulerian and Lagrangian velocities coincide at the same point of the fiber bundle $\mathcal{F}$, they are vector fields in different spaces. The Eulerian velocity is a vector space defined on the standard fiber manifold $F$. Indeed, because the fiber at any point $F_{(x, t)}$ is diffeomorphic with the standard fiber $F$, according to the minimal constrains, we can map vectors tangent to any fiber, into vectors tangent to the standard fiber $F=M$. So, a cross-section $\sigma$ in $\mathcal{F}$ generates a vector field on $F$ at any moment of time, the Eulerian flow. The integral curves of this field are, at every moment of time, the collections of time-dependent stream lines, they lie in the standard fiber, and they have no special assigned parameter. Contrary to the stream lines, the path lines are time-parametrized, hence constant, and they lie in the fiber bundle. Again, the collection of path lines do not coincide with the flow net in general (they coincide if the flow is stationary). It is also true that the path lines never cross the flow net lines.

Since $\sigma\left(M, t_{0}\right) \simeq M$ is a diffeomorphisms because of the minimal constraints, the image of any compact set in $M$ is a compact set in $F_{(x, t)}$. Such sets are the particle structures that remain "stable" to this extent. If such a set is a submanifold of dimension 1, we call it particle line, or material line, or circuit lines, or filament. Once identified in the reference fluid container, this line conserves its topological proprieties in time. If the submanifold is 2-dimensional, it is a particle surface, or free fluid surface, etc., and so on. We noticed above that the particle paths are restrictions of the cross-sections describing the dynamics for constant $x$. Similarly, particle lines are restrictions of the cross-section for constant time, and on subsets of the $M$ manifold: $\left.\sigma(x, t)\right|_{\left(x \in D, t=t_{0}\right)}=\left.\hat{\sigma}(x)\right|_{x \in D}$.

There is another interesting approach about the path lines as orbits of a group of diffeomorphisms of the spatial part of the base space. Actually, any such diffeomorphism (any 


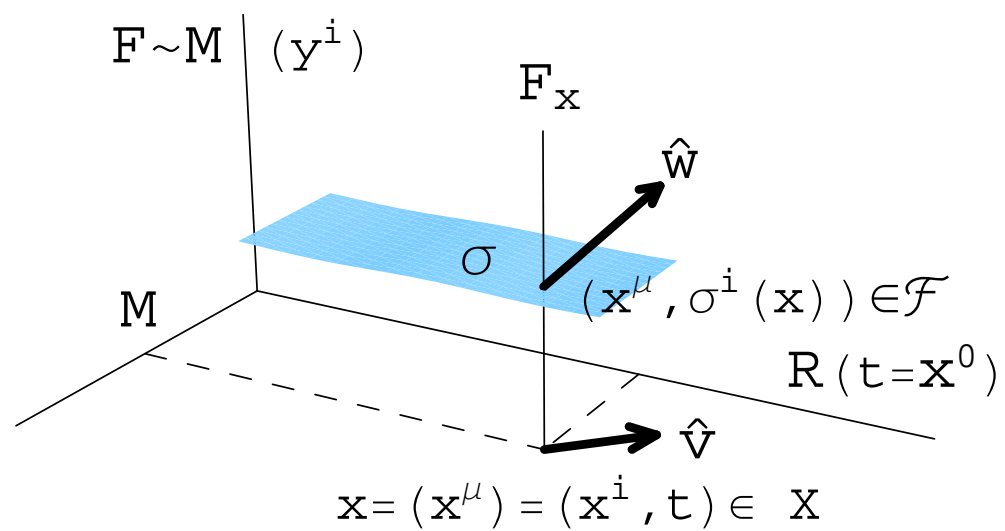

Figure 3: Structure of the fiber bundle associated with a fluid.

flow) can be understood as a relabeling operation of the fluid particles. Such a relabeling operation is connected with a continuous symmetry of the system. If we consider the fluid a Lagrangian system, and the flow is incompressible, the Noether current associated to this symmetry is the fluid momentum conservation.

In the following, we give an interpretation of the transformation between variation of Eulerian and Lagrangian quantities in terms of a connection. Let us consider again the fiber bundle $\mathcal{F}$ representing a fluid confined in a fixed space domain identified by the manifold $M \ni\left(x^{i}\right)$, where $i, j=1, \ldots, 3$ and $\mu=0, \ldots, 3$. The base space is the direct product $X=M \times \mathbf{R} \ni\left(x^{\mu}\right)=\left(x^{i}, x^{0}=t\right)$. We choose the fiber $F=M$, a trivial identity structure group $G=\{e\}$, the projection $\Pi, F_{x}=\Pi^{-1}(x)$ and a cross-section $\sigma: X \rightarrow \mathcal{F}$. The cross-section maps $x=\left(x^{\mu}\right) \rightarrow \sigma^{a}=\left(x, \sigma^{j}(x)\right)$, and its differential $d \sigma: T X \rightarrow T \mathcal{F}$ $\operatorname{maps} T_{x} X \ni \hat{v}(x)=\left(\vec{v}, v^{0}\right)=\left(v^{i}, v^{0}\right)=\left(v^{\mu}\right) \rightarrow \hat{w}=\left(\vec{w}, w^{0}, \bar{w}\right)=\left(w^{i}, w^{0}, \bar{w}^{j}\right) \in T_{\sigma(x)} \mathcal{F}$, with $a=(\mu, j)$. In components, the action of the differential, which is a section in the first jet fiber bundle over $\mathcal{F}$, reads

$$
\begin{aligned}
& d \sigma(\hat{v})=\left(\frac{\partial \sigma^{a}}{\partial x^{\mu}} v^{\mu}\right)=\left(\frac{\partial \sigma^{\nu}}{\partial x^{\mu}} v^{\mu}, \frac{\partial \sigma^{j}}{\partial x^{\mu}} v^{\mu}\right)=\left(\frac{\partial x^{\nu}}{\partial x^{\mu}} v^{\mu}, \frac{\partial \sigma^{j}}{\partial x^{i}} v^{i}+\frac{\partial \sigma^{j}}{\partial t} v^{0}\right)= \\
& \left(v^{\nu},\left(v^{i} \frac{\partial}{\partial_{i}}\right) \sigma^{j}+v^{0} \frac{\partial \sigma^{j}}{\partial t}\right)=\left(\vec{v}, 1,(\vec{v} \cdot \nabla) \vec{\sigma}+v^{0} \frac{\partial}{\partial t} \vec{\sigma}\right) .
\end{aligned}
$$

If we restrict ourselves on curves being path lines in the time parametrization, the tangent vectors are $\hat{v}=(\vec{v}, 1)$, i.e. $v^{0}=1$. The interpretation of Eq. (2.1) is as follows. Spatial part $\vec{\sigma}$ of vectors in the tangent space to the base are in one-to-one correspondence with vectors in the tangent space to the fiber, by the triviality of $\mathcal{F}$. So $\vec{\sigma}$ is actually a fiber vector, that is an "Eulerian" vector in a local space frame. This Eulerian vector is mapped to a vector in the tangent space to the bundle, which is a "Lagrangian" vector.

$$
T M \ni \vec{v} \rightarrow\left[(\vec{v} \cdot \nabla)+\frac{\partial}{\partial t}\right] \vec{\sigma}, \text { with } \hat{\sigma}=(x, \vec{\sigma}) \in T \mathcal{F} .
$$


If we put $\vec{v}_{E}=\vec{\sigma}$, Eq. (2.2) reads $d \sigma\left(\vec{v}_{E}\right)=\vec{v}_{L}$, that is the well known transformation between the partial time derivative and the material (total) derivative, Eq. (1.3). In this sense, Eq. (2.2) describes a connection in $\mathcal{F}$ in the first jet bundle $J^{1}$ (for example see Olver's book [5]). Coming down to the $\mathcal{F}$ bundle we note that the only possible connection is a trivial one, with zero coefficients. This is because the bundle is trivial, so the only admissible infinitesimal transformations are translations. The situation is different if the shape of the fluid container is allowed to change in time.

If the shape of the reference fluid container changes with time (boundaries not fixed anymore) the fiber $F_{x}$ depends on the point $\left(x^{i}, t\right) \in X$ through the time dependence and the bundle is not anymore a global Cartesian product. Consequently, it has a non-trivial structure group $G$.

We consider the fluid "drop" as a connected, simple-connected domain $\mathcal{D}_{\Sigma} \simeq D_{3} \subset \mathbf{R}^{3}$ with smooth boundary (shape) $\partial \mathcal{D}=\Sigma$, and under no external forces or torques. By $\simeq D_{3}$ we mean a diffeomorphisms with the 3 -dimensional disc $x^{2}+y^{2}+z^{2}<=1$. The drop has a set of possible shapes. If we can parametrize the set of all possible shapes with coordinates, we could set the structure of a manifold $M$. The shape coordinates can be determined by the expansion in spherical harmonics, for example, and we can associate to $M$ the $l_{2}(\mathbf{C})$ space structure with the topology induced by the norm. We call $M$ the shape space of the drop. The base space will be, like in the previous case, $X=M \times \mathbf{R} \ni(\Sigma, t)$.

For any shape we choose a trihedron fixed in this shape, for example the origin in the center of mass, and the axes directed towards the positions of some chosen zeros of the spherical harmonics. The configuration of the fluid within the given shape $\Sigma$ will be referred to this trihedron. For a given shape $\Sigma$, all possible configurations of the fluid particles $\left\{\vec{r} \mid \vec{r} \in \mathcal{D}_{\Sigma}\right\}$ can be described by the set of diffeomorphic (shape invariant) transformation of $\mathcal{D}_{\Sigma}$ onto itself. These transformations form a Lie group of diffeomorphisms Diff $\Sigma$. Any element $g_{\Sigma}$ of this group maps some distribution of particles inside this shape into another distribution of particles within the same shape. So, by the minimal constrains, the fiber over $x=(\Sigma, t) \in M$ is represented by the group of diffeomorphisms of the shape $\Pi^{-1}(\Sigma, t)=$ Diff $_{\Sigma}$. The structure group is the group of diffeomorphisms of the 3-dimensional disc, Diff $D_{3}$, which is the group model for all the other diffeomorphisms groups. Consequently, $\mathcal{F}$ is a principal bundle, and the coordinate on the fiber over $(\Sigma, t)$ is a certain group transformation $\operatorname{Diff}_{\Sigma} \ni g_{\Sigma}: \mathcal{D}_{\Sigma} \rightarrow \mathcal{D}_{\Sigma}$.

This construction must be carried out for all possible shapes. Thus, the total configuration space of the fluid $\mathcal{F}$ is a fiber bundle over the base $X$, of fiber $D i f f_{\Sigma}$. A shape evolution will be identified by a (time-like) curve $\gamma \in X$, that is a regular curve of shapes $\Sigma(t)$ parametrized by time. For any particular shape we have to integrate a set of dynamical equations $\triangle(\Sigma, \vec{r}, t)$ in order to find the positions of the particles associated to that shape. The shape at any moment of time determines the position of particles within the fiber. Hence, a cross-section $\sigma: X \rightarrow \mathcal{F}$ represents the evolution of the drop, namely in components $t \rightarrow \Sigma(t) \rightarrow \vec{r}_{L}\left(\vec{r}_{0}, t\right)=g_{\Sigma(t)}\left(\vec{r}_{0}\right)$. From the geometrical point of view, the dynamical equations of the free surface fluid are equations for this section. These are basically the equation of continuity, equations for momentum conservation (Euler equations) and energy transfer equation.

For any shape in $M$ we need to specify its fixed reference trihedron, and its reference (we may call it initial) distribution of particles $\vec{r}_{0}$. This choice is not unique, and the freedom involved is a typical gauge freedom. 
Translation of the drop center of mass could be eliminated from the beginning, but the shapes should also conserve total angular momentum. Angular momentum can be changed by deformations (motion in the base space) and also by particle rotations (motions in the fiber). We need to "synchronize" the succession of deformations with a unique succession of rotations, such that total angular momentum to be constant. In that, we can introduce a new type of connection, different from that one introduced above between Eulerian and Lagrangian approach on tangent vectors Eq. (2.2).

For any given smooth curve $\gamma$ in the base space $M$, we need to lift it to a curve $\gamma^{\prime}$ in the total space $\mathcal{F}$ in a unique way. Remember that a lift is a map $\gamma \in M \rightarrow \gamma^{\prime} \in \mathcal{F}$ such that $\Pi\left(\gamma^{\prime}\right)=\gamma$. However, the lift of a path is not unique by definition. The mathematical tool needed to make it unique is the connection. A connection, or better said its differential expression, would assign to any tangent vector $\vec{v}(x) \in T_{x} M$ an element in $T F_{x}$, which is the Lie algebra of the group Diff $\Sigma$. Globally, when we move along a closed path in $M$ the corresponding lifted path in $\mathcal{F}$ may not be closed. That is for $\gamma\left(x_{0}\right)=\gamma x_{1}$ we may have $\gamma^{\prime}\left(x_{0}\right)=\gamma^{\prime} x_{1}$ in $F$. Two different points on the same fiber mean a relabeling of the particles, or a motion inside the drop. Such a relabeling could be associated with a finite non-zero rotation of the drop. The drop begins to move by changing its shape and ends up to the same initial shape after a finite amount of time. But during this motion, it actually undergoes a net rotation.

A similar situation happens when we build the configuration space of a deformable body. Again, we choose for any shape a trihedron fixed in this shape. The orientation of the body, ignoring free translations of the center of mass, could be described by a proper rotation matrix $\hat{R} \in S O(3)$ which maps the body fixed trihedron to a space frame contained in the ambient space in which the drop is constrained to move, i.e. $\mathbf{R}^{3}$. Thus, the total configuration space $\mathcal{F}$ is a fiber bundle over the base $M \times \mathbf{R}$, of fiber $S O(3)$.

Like in the case of the drop, the angular momentum of the body can be changed by deformations (motion in the base space) and also by rotations (motions in the fiber). In this example, the connection assigns to any tangent vector $\vec{v}(x) \in T M$ an element in $T S O(3)$, which is nothing but the Lie algebra so(3). When we move along a closed path in $M$ the corresponding lifted path is not closed in general. Two different points on the same fiber mean a change in the orientation, a rotation. The body moves and changes its shape, but during this motion, it undergoes a rotation. However, because the $S O(3)$ Lie group is not commutative there are problems in integrating this lifted path in the fiber.

\section{Path lines, stream lines and particle contours}

We discus here only finite time flows with $t \in\left[t_{1}, t_{2}\right],-\infty<t_{1}<t_{2}<\infty$. We begin our construction with the fluid initial reference container, i.e. a domain $D_{0} \subset \mathbf{R}^{3}$. We construct the base space $X=\mathbf{R}^{3} \times\left[t_{1}, t_{2}\right]$, and we assign a local coordinate system in $\vec{r}_{0} \in$ $D_{0}$. We assume we are given the fluid flow as smooth homeomorphisms $\vec{r}_{L}: D_{0} \times\left[t_{1}, t_{2}\right] \rightarrow$ $\mathbf{R}^{3}$ such that the restriction $\left.\vec{r}_{L}\right|_{D_{0} \times\{t\}}$ is injective for any fixed $t \in\left[t_{1}, t_{2}\right]$. In coordinates this reads $\left(\vec{r}_{0}, t\right) \rightarrow \vec{r}_{L}\left(\vec{r}_{0}, t\right)$. The family of curves $L=\left\{\gamma_{L} \Rightarrow \vec{r}_{L}\left(\vec{r}_{0}, t\right) \mid \vec{r}_{0} \in D_{0}\right\}$ is the particle paths, with tangents $\dot{\vec{r}}_{L}=\vec{v}_{L}$ and metric $g_{L}=v_{L}^{2}$. These curves can be lifted in the base space and mapped into a family $\tilde{L}=\left\{\gamma_{L}^{\prime} \Rightarrow\left(\vec{r}_{L}\left(\vec{r}_{0}, t\right), t\right) \in \mathbf{R}^{3} \times\left[t_{1}, t_{2}\right] \mid \vec{r}_{0} \in D_{0}\right\}$. The metric of $\gamma_{L}^{\prime}$ is $\tilde{g}_{L}=v_{L}^{2}+1$. Both $\gamma_{L}$ and $\gamma_{L}^{\prime}$ are Lagrangian path lines viewed in 
different spaces.

For any $t \in\left[t_{1}, t_{2}\right]$ we can construct $D_{t}=\vec{r}_{L}\left(D_{0}, t\right) \subset \mathbf{R}^{3}$. A particle contour is a parametrized curve $\Gamma_{0}=\left\{\gamma_{0}(s) \subset D_{0}, s \in I\right\} \subset D_{0}$. The question is what happens to such a particle contour in time. Is $\Gamma_{t}=\left\{\gamma(s, t)=\vec{r}_{L}\left(\gamma_{0}(s), t\right)\right\}$ a regular curve with the same topology as $\Gamma_{0}$ ? We have the following result

Theorem 1. The set $\Gamma_{t}$ defined by $\gamma(s, t)$ as above is a regular parametrized curve if

$$
\left.\hat{J}\left(\vec{r}_{L}\left(\vec{r}_{0}, t\right)\right)\right|_{t=\text { const. }} \cdot \vec{t}_{\Gamma_{0}} \neq 0,
$$

for $\forall s \in I, t \in\left[t_{1}, t_{2}\right]$. Here $\vec{t}$ is the tangent vector to a curve.

Proof. We have

$$
\frac{\partial \vec{r}}{\partial s}(s, t)=\frac{\partial x_{L}^{i}}{\partial \gamma^{j}} \frac{d \gamma^{j}}{d s}=\frac{\partial x_{L}^{i}}{\partial x_{0}^{j}} \cdot \vec{t}_{\Gamma_{0}}^{j}(s),
$$

which represents the requested inequality.

In other words, a particle contour at the initial moment of the flow remains a regular curve while transported by the flow in time if the unit tangent of this initial curve is not in the kernel of the Jacobian matrix of the Lagrangian paths function of the initial coordinates (the flow). If conditions in Theorem 1 are fulfilled the particle contour $\Gamma_{0}$ remains a regular curve during the flow, so one can apply circulation or other types of theorems on it.

The question is if the set $\cup_{t \in\left[t_{1}, t_{2}\right]} D_{t}$ is a submanifold of $\mathbf{R}^{3}$ ? If it is, we can assign local coordinates for its points in the form $p=\left(\vec{r}_{0}, t\right)$. In other words, if the reunion of all path lines over a certain finite interval of time is dense enough to form a topological space. The answer can be given at least locally, by using the flow box theorem. Obviously, the Lagrangian velocity field of any particle $\vec{v}_{L}$ fulfils the conditions for the existence of flow boxes on $X$. Indeed, for any $\left.t \in\left[t_{1}, t_{2}\right]\right]$, and any point $p=(\vec{r}, t) \subset D_{t}$ we can find a neighborhood $V(\vec{r})$ and $t \pm \delta t$ such that it exists $a>0$ and the triple

$$
\left((V(\vec{r}),(t-\delta t, t+\delta t)) a, \gamma_{L}\left(\vec{r}_{L}\left(\vec{r}_{0}, t\right), t+\lambda\right)\right),
$$

is a flow box.

Moreover, we assume that the fluid flows in such a way that $X$ is a topological space with the product topology of $\mathbf{R}^{3} \times \mathbf{R}$. We also assume that the fluid flows in a bounded region (bounded fixed region or free compact surface), so the Lagrangian velocity field has compact support in $X$. Consequently $\gamma_{L}\left(\vec{r}_{0}, t\right)$ are maximal integral curves and form a foliation of $X$. Since the field of velocities of particles has compact support, it is complete, and any of its integral curves can be extended so that its domain of parameter becomes $\mathbf{R}$. So the Lagrangian paths $\gamma_{L}\left(\vec{r}_{0}\right)$ form a foliation of the manifold $D_{t}$ which is homeomorphic with $D_{0}$. We mention again that inside each $D_{t}$ we have $\vec{v}_{E}\left(\vec{r}_{L}\left(\vec{r}_{0}, t\right), t\right) \equiv \dot{\vec{r}}_{L}\left(\vec{r}_{0}, t\right)$, but inside the same $D_{t}$ the integral curves of $\dot{\vec{r}}_{L}$ are not the $\gamma_{L}$ curves.

There are of course differences and similarities between the stream and path lines. In Fig. 4 we present a cross section into a spherical drop of incompressible inviscid fluid in oscillation with an $l=2$ mode. The thin lines are the stream lines and the thick line is a path line. 


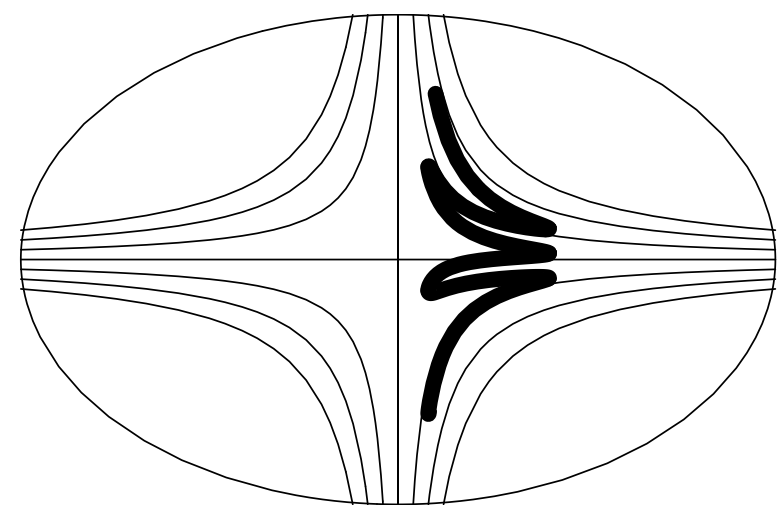

Figure 4: Cross section into a spherical drop of incompressible inviscid fluid in oscillation in a $\mathrm{l}=2$ mode. The thin curves are the stream lines, while the thick curve is an example of a path line.

Establishing a Lagrangian-Eulerian change of frames in lower dimensional flows is not quite trivial. In order to introduce Eulerian coordinates on a moving curve, for example a thin vortex filament in motion, we consider that the Lagrangian coordinates along a curve of length $L$ are given by the arc-length parametrized form of the curve $\vec{r}(s, t)$. The curve is in motion, and the velocity can be expressed in its Serret-Frenet local frame $\{\vec{t}, \vec{n}\}$ in the form $\vec{V}(s, t)=U(s, t) \vec{n}+W(s, t) \vec{t}$ We introduce the mapping $e:[0, L] \rightarrow \mathbf{C}$

$$
e(s, t)=\int^{s} e^{i \theta\left(s^{\prime}, t\right)} d s^{\prime}
$$

from the Lagrangian coordinate to the Eulerian one, where $\theta=\int^{s} \kappa\left(s^{\prime}, t\right) d s^{\prime}$ is the tangent angle of the curve, and $\kappa$ is its curvature. In the Eulerian coordinate we can express all the intrinsic properties of the curve,namely $\theta=-i \ln \left(e_{s}\right), \kappa=-i e_{s s} / e_{s}$, and the dynamics of the transformation of coordinates is given by $e_{s t}=\left[(W-i U) e_{s}\right]_{s}[6]$. In terms of the new coordinate $e$ and time the dynamical equation for the velocity components is $\theta_{t} e^{i \theta}=e^{2 i \theta}(W-i U)_{e}$. Let us choose now a curve motion with zero normal velocity and constant tangential velocity. Since such a motion is only a re-parametrization of the curve, i.e it is not a real motion, we expect the Eulerian coordinate to remain constant. Indeed, from the above relations we have $e_{s t}=0$ so $e=$ const.

\section{Application: solitary waves and path lines for a two- dimensional liquid drop}

Nonlinear oscillations of a liquid drops introduce new phenomena and more complicated patterns (higher resonances, solitons, compactons, breakup, fragmentation, fractal structures, superdeformed shapes) than cannot be described by a linear theory. There are experiments [7] where special rotational patterns of circulation emerge: a running wave originates on the surface of the drop and then propagates inward. The dynamics governing one-dimensional surface oscillations of a perfect ( $\rho=$ const.), irrotational fluid drop (or bubble, shell) can be described by the velocity potential $\Phi(\vec{V}=\nabla \Phi)$ and a corresponding Hamiltonian [8]. By expanding the Hamiltonian and dynamical equations in terms of a small parameter, i.e. the amplitude of the perturbation $\eta$ over the radius of drop $R_{0}$, the 
usual linear theory is recovered in the first order. Higher order non-linear terms introduce deviations and produce large surface oscillations like cnoidal waves $[9,10]$. These oscillations, under conditions of a rigid core of radius $R_{0}-h$ and non-zero angular momentum, transform into solitary waves.

The total hydrodynamic energy $E$ consists of the sum of the kinetic $T$ and potential $U$ energies of the liquid drop. The shape function is assumed to factorize, $r(\theta, \phi, t)=$ $R_{0}(1+g(\theta) \eta(\phi, t))$. All terms that depend on $\theta$ are absorbed in the coefficients of some integrals and the energy reduces to a functional of $\eta$ only. The potential energy is given by the surface energy $U_{S}=\left.\sigma\left(\mathcal{A}_{\eta}-\mathcal{A}_{0}\right)\right|_{V_{0}}$, where $\sigma$ is the surface pressure coefficient, $\mathcal{A}_{\eta}$ is the area of the deformed drop, and $\mathcal{A}_{0}$ the area of the spherical drop, of constant volume $V_{0}$. The kinetic energy $T=\rho \oint_{\Sigma} \Phi \nabla \Phi \cdot d \vec{S} / 2$, the kinematic free surface boundary condition $\Phi_{r}=\partial_{t} r+\left(\partial_{\theta} r\right) \Phi_{\theta} / r^{2}+\left(\partial_{\phi} r\right) \Phi_{\phi} / r^{2} \sin \theta$, and the boundary condition for the radial velocity on the inner surface $\left.\partial_{r} \Phi\right|_{r=R_{0}-h}=0$, result in the expression

$$
T=\frac{R_{0}^{2} \rho}{2} \int_{0}^{\pi} \int_{0}^{2 \pi} \frac{R_{0} \Phi \eta_{t} \sin \theta+\frac{1}{R_{0}} g \eta_{\phi} \Phi \Phi_{\phi}(1-\sin \theta)}{\sqrt{1+g_{\theta}^{2} \eta^{2}+g^{2} \eta_{\phi}^{2}}} d \theta d \phi .
$$

If the total energy, written in the second order in $\eta$, is taken to be a Hamiltonian $H[\eta]$, the time derivative of any quantity $F[\eta]$ is given by $F_{t}=[F, H]$. Defining $F=\int_{0}^{2 \pi} \eta(\phi-V t) d \phi$ it results

$$
\frac{d F}{d t}=\int_{0}^{2 \pi} \eta_{t} d \phi=\int_{0}^{2 \pi}\left(2 C_{2} \eta_{\phi}+6 C_{3} \eta \eta_{\phi}-2 C_{4} \eta_{\phi \phi \phi}\right) d \phi=0,
$$

which leads to the $\mathrm{KdV}$ equation. Here $\mathcal{C}_{2}=\sigma R_{0}^{2}\left(S_{1,0}^{1,0}+S_{0,1}^{1,0} / 2\right)+R_{0}^{6} \rho V^{2} C_{2,-1}^{3,-1} / 2$, $\mathcal{C}_{3}=\sigma R_{0}^{2} S_{1,2}^{1,0} / 2+R_{0}^{6} \rho V^{2}\left(2 S_{-1,2}^{3,-1} R_{0}+S_{-2,3}^{5,-2}+R_{0} S_{-2,3}^{6,-2}\right) / 2, \mathcal{C}_{4}=\sigma R_{0}^{2} S_{2,0}^{-1,0} / 2$, with $S_{i, j}^{k, l}=$ $R_{0}^{-l} \int_{0}^{\pi} h^{l} g^{i} g_{\theta}^{j} \sin ^{k} \theta d \theta$. Terms proportional to $\eta \eta_{\phi}^{2}$ can be neglected since they introduce a factor $\eta_{0}^{3} / L^{2}$ which is small compared to $\eta_{0}^{3}$, i.e. it is in the third order. Therefore, the energy of the non-linear liquid drop model can be interpreted as the Hamiltonian of the one-dimensional KdV equation. The coefficients in Eq. (4.2) depend on two stationary functions of $\theta$ (the depth $h(\theta)$ and the transversal profile $g(\theta)$ ), hence, under the integration, they involve only a parametric dependence.

The KdV equation has the following cnoidal wave (Jacobi elliptic function) as exact solution

$$
\eta=\alpha_{3}+\left(\alpha_{2}-\alpha_{3}\right) s n^{2}\left(\sqrt{\frac{C_{3}\left(\alpha_{3}-\alpha_{2}\right)}{12 C_{4}}}(\phi-V t) \mid m\right),
$$

where $\alpha_{1}, \alpha_{2}, \alpha_{3}$ are constants of integration, $m^{2}=\left(\alpha_{3}-\alpha_{2}\right) /\left(\alpha_{3}-\alpha_{1}\right)$. This solution oscillates between $\alpha_{2}$ and $\alpha_{3}$, with a period $T=2 K(m) \sqrt{\frac{\left(\alpha_{3}-\alpha_{2}\right) C_{3}}{3 C_{4}}}$, where $K(m)$ is the period of a Jacobi elliptic function $\operatorname{sn}(x \mid m)$. The parameter $V$ is the velocity of the cnoidal waves and $\alpha_{1}+\alpha_{2}+\alpha_{3}=\frac{3\left(V-C_{2}\right)}{2 C}$. In the limit $\alpha_{1}=\alpha_{2}=0$ the solution Eq. (4.3) approaches

$$
\eta=\eta_{0} \operatorname{sech}^{2}\left[\sqrt{\frac{\eta_{0} C_{3}}{12 C_{4}}}(\phi-V t)\right]
$$



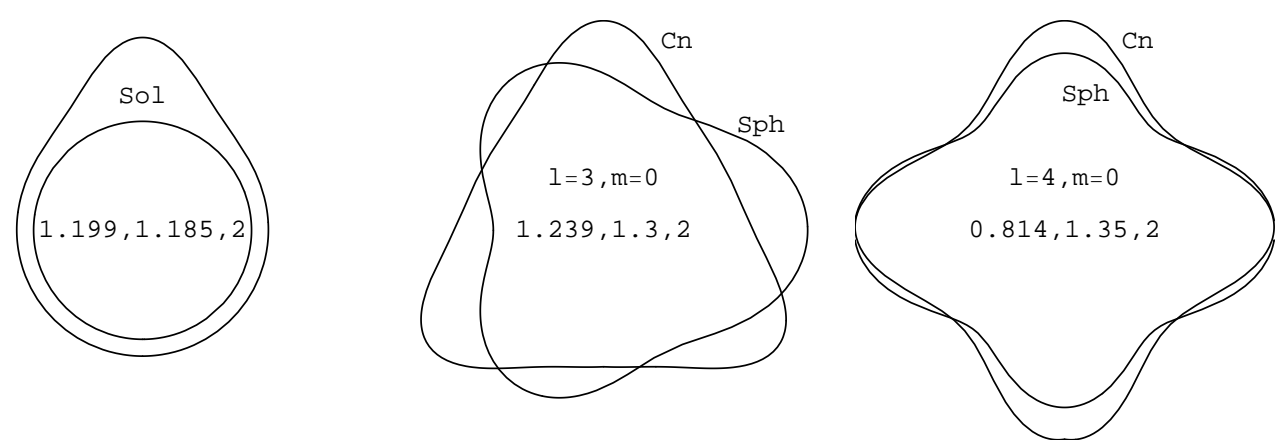

Figure 5: Nonlinear modes in a two-dimensional drop.

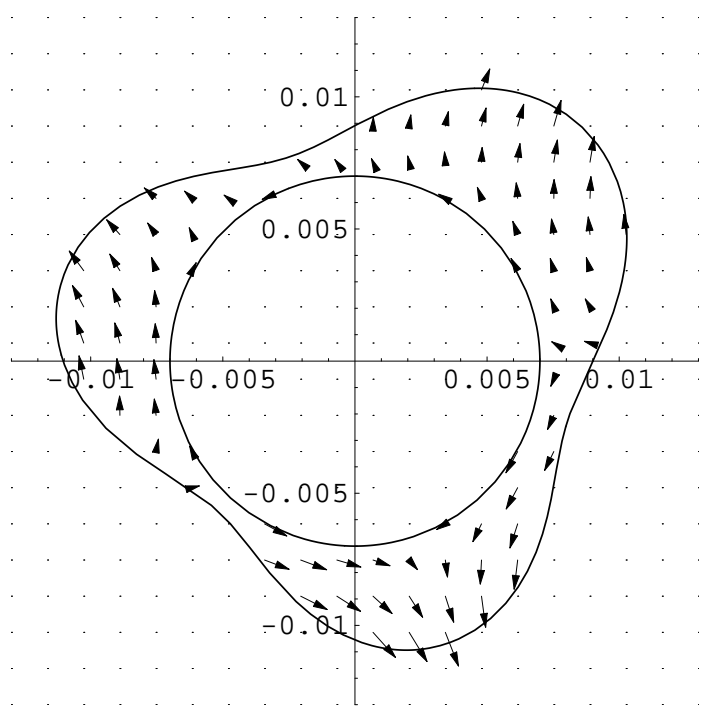

Figure 6: Eulerian velocity field in a cnoidal wave.

which is the soliton solution of amplitude $\eta_{0}$. Small oscillation occur when $\alpha_{3} \rightarrow \alpha_{2}$ and $m \rightarrow 0, T \rightarrow \pi / 2$. Consequently, the system has two limiting solutions, a periodic and a localized traveling profile, which deform one into the other, by the initial conditions and the velocity parameter $V$, Fig.5. The cnoidal solution Eq. (4.3) depends on the parameters $\alpha_{i}$ subjected to the volume conservation and the periodicity condition of the solution (for the final soliton state this condition should be taken as a quasi-periodicity realized by the rapidly decreasing profile). The periodicity restriction reads

$$
K\left(\sqrt{\frac{\alpha_{3}-\alpha_{2}}{\alpha_{3}-\alpha_{1}}}\right)=\frac{\pi}{n} \sqrt{\alpha_{3}-\alpha_{1}}, \quad n=1,2, \ldots, 2 \sqrt{\alpha_{3}-\alpha_{1}} .
$$

Hence, a single free parameter remains, which can be taken either one out of the three $\alpha$ 's, $V$ or $\eta_{0}$. In order to find the velocity filed, and consequently to integrate the corresponding Hamiltonian system to obtain the particles path, we have to calculate the velocity 
potential. Following the procedure in [9] we expand the potential as a power series

$$
\Phi=\sum_{n=0}^{\infty}\left(\frac{r}{R_{0}}-1\right)^{n} f_{n}(\theta, \varphi, t) .
$$

The potential fulfils the Laplace equations plus boundary conditions at the rigid core surface and at the free surface which generate recursion relations for all the unknown functions $f_{n}$ depending only on $f_{0}$, which reads

$$
\frac{\partial f_{0}}{\partial \varphi}=\frac{V R_{0}^{3} \sin ^{2} \theta \eta(1+2 \eta)}{h} .
$$

With $\eta$ given by Eq. (4.3), and appropriate choice for the angular velocity $V$ of the cnoidal wave shape, the depth $h$ of the boundary layer we obtain the velocity potential, and through its derivatives the local velocity field. In Fig. 6 we present such a velocity field, in the Eulerian frame for a cnoidal wave with period $\pi / 3$.

\section{Conclusions}

In the present paper we introduced a fiber bundle differential geometry formalism for the stream, streak and path lines in a fluid, for both fixed container and free surface. As a direct application we study the dynamics of a two-dimensional liquid drop with surface tension, from the Hamiltonian point of view. Cnoidal and solitary surface waves are obtained as solutions for this system. The Eulerian velocity field shows motion of the particles up and down and forward-backward pretty much like in a Stokes wave [11] (see $[10,12]$ for the case of solitary waves). The problem of particle paths under a soliton profile on a circle will be addressed in a forthcoming paper. In the direction of extension of the framework presented in this paper towards other nonlinear hydrodynamics configurations, we would like to include two nonlinear wave systems that have been exactly solved: the Gerstner's solution for the classical deep water wave, [13] (see also a recent discussion about this problem in [14]), and the edge wave solution [15]. These systems are the only ones having explicit solutions for the nonlinear governing equations for gravity water waves with a non-flat free surface.

Acknowledgments. The author thanks Northwestern State University for the sabbatical support. The assistance from NSU's Enrichment Fund Grant Program NEF, Agreement PD 07-08 R1-005 is also gratefully acknowledged.

\section{References}

[1] ArIs R, Vectors, Tensors, and the Basic Equations of Fluid Mechanics, (Dover Publ., New York 1989)

[2] Lamb H, Hydrodynamics, (Dover Publ., New York 1932)

[3] Marsden J E, Pekarsky S, Shkoller S, and West M, Variational methods, multisymplectic geometry and continuum mechanics, J. Geom. Phys. 38 (2001), 253-284. 
[4] Marsden J E, and Shkoller S, Multisymplectic geometry, covariant Hamiltonians, and water waves, Math. Proc Cambridge Philos. Soc. 125 (1999) 553-575.

[5] Olver P J, Applications of Lie Groups to Differential Equations, 1st edn (Springer, Berlin Heidelberg New York 1986).

[6] Kawamoto S, An Exact Transformation from the Harry Dym Equation to the Modified KdV Equation, J. Phys. Soc. Jpn. 54 (1985), 2055-2056.

[7] Trinh E, and WAng T G, Large-amplitude free and driven drop-shape oscillations: experimental observations, J. Fluid Mech. 122 (1982), 315-338.

[8] Tsamopoulos J A, and Brown R A, Nonlinear oscillations of inviscid drops and bubbles, J. Fluid Mech. 127 (1983), 519-537.

[9] Ludu A, and Dranyer J P, Nonlinear Modes of Liquid Drops as Solitary Waves, Phys. Rev. Lett 80 (1998), 2125-2128.

[10] Ludu A, Nonlinear Waves and Solitons on Contours and Closed Surfaces, (Springer-Verlag, Heidelber 2007).

[11] Constantin A, The trajectories of particles in Stokes waves, Invent. Math. 166 (2006), 523-535.

[12] Constantin A, and Escher J, Particle trajectories in solitary water waves, Bull. Amer. Math. Soc. 44 (2007), 423-431.

[13] Gerstner F., Theorie der Wellen samt einer daraus abgeleiteten Theorie der Deichprofile, Ann. Phys. 2 (1809) 412-445.

[14] Constantin A, On the deep water wave motion, J. Phys. A 34 (2001) 1405-1417.

[15] Constantin A, Edge waves along a sloping beach, J. Phys. A 34 (2001) 9723-9731. 